\title{
Expansion of the Inhomogeneous Modular Transformations with Modular Group Properties
}

\author{
İsmet YILDIZ ${ }^{*}{ }^{*}$, Kezban ALTUN ${ }^{1}$, Betül ÖZTÜRK ${ }^{1}$ (๑) \\ ${ }^{1}$ Department of Mathematics, Faculty of Science and Art, Duzce University, Düzce, Turkey \\ Geliş / Received: 13/05/2021, Kabul / Accepted: 17/07/2021
}

\begin{abstract}
In this study, inhomogeneous modular groups and their basic properties are examined, and the relationships between homogeneous and inhomogeneous linear transformation concepts are discussed. The definition of the modular group is demonstrated by using inhomogeneous linear transformations. With the help of modular group conditions, the upper half-plane is defined with the help of the images under the elements of the modular group $\Gamma$ and the formation of inhomogeneous modular transformations on different transformations is examined. In addition, the theorem about the cases of the modular group has been determined with a distinct method.
\end{abstract}

Keywords: Inhomogeneous linear transformation, Complex numbers, Modular group.

\section{Modüler Grup Özellikleri İle İnhomojen Modüler Grupların Genişlemesi}

\section{Öz}

Bu çalışmada inhomojen modüler gruplar ve onların temel özellikleri incelenerek homojen, inhomojen lineer dönüşüm kavramları arasındaki ilişkiler ele alınmıştır. Inhomojen lineer dönüşümler kullanılarak modüler grubun tanımı gösterilmiştir. Modüler grup koşulları yardımı ile $\Gamma$ modüler grubunun elemanları altındaki görüntüleri yardımıyla üst yarı düzlem tanımlanıp inhomojen modüler dönüşümlerin farklı dönüşümler üzerine oluşturulması incelenmiştir. Ayrıca, modüler grubun durumları ile ilgili teorem değişik bir yöntemle kanıtlanmıştır.

Anahtar Kelimeler: İnhomojen doğrusal dönüşüm, Karmaşık sayılar, Modüler grup.

\section{Introduction}

Henry Poincare first demonstrated some important findings that could serve as a basis for discrete group theory towards the end of the nineteenth century, and these were used in the generalization of elliptic functions theory. Many scientists investigated the functions left invariant by these discrete groups, which were named Fuchsian groups after Henry Poincare's systematic work on them. Due to their suitability for topologic group structure, linear fractional transformation groups acquired a special significance with the discovery of NonEuclidean geometry and invariant theory in the 19th century, and they were extensively studied by analysis and algebraic methods.

In recent years, it has become clear that congruence subgroups of the $\Gamma$ modular group, that is $\Gamma(N), \Gamma_{0}(N)$ and $\Gamma_{1}(N)$, have been extensively studied due to their significance in elliptic curves, integral quadratic forms, and elliptic modular functions. 
In this study, firstly, homogeneous and inhomogeneous transformations will be defined and their important properties will be given. Further, the modular group is defined using these transformations. After these, modular transformations are classified by means of the transformations and also generators of the modular group and their relations are given. Furthermore, the fundamental region is defined. Tessellation upper half plane is explained because of existence of the fundamental region.

For linear inhomogeneous transformation

$$
\begin{gathered}
\varphi: \mathbb{C}^{*} \rightarrow \mathbb{C}^{*} \\
z \rightarrow w=\varphi(z)=\frac{a z+b}{c z+d}
\end{gathered}
$$

and

$$
a d-b c \neq 0 \text { where } a, b, c, d \in \mathbb{C} \text {. }
$$

The inhomogeneous linear transformation is reversible and preserves the $\mathbb{C}^{*}$ sphere for this transformation. The inhomogeneous modular transformation is represented by $\Psi$.

When $\varphi \in \psi$ is not identical, it has at most two fixed points and $\varphi$ transform with respect to these points is classified as hyperbolic, elliptic, loxodromic and parabolic transformations.

If homogeneous modular transformations whose elements are rational integers and the 2-by-2 integral matrices with determinant equal to 1 then they are homogeneous linear transformations. The group formed by homogeneous modular transformations is called homogeneous modular group.

The generations of inhomogeneous modular transformations expanded by reflections were investigated in the analysis of modular group conditions, and a theorem concerning modular group generations was proved using a distinct approach. Also, the fundamentals of inhomogeneous modular transformations were developed using newly identified transformations.

\section{Material and Methods}

\section{Inhomogeneous Linear Transformation}

The field of real numbers, complex numbers and Riemann sphere are denoted by $\mathbb{R}, \mathbb{C}$ and $\mathbb{C} *$ respectively. The following is the definition of an inhomogeneous linear transformation.

$$
\begin{gathered}
\varphi: \mathbb{C}^{*} \rightarrow \mathbb{C}^{*} \\
z \rightarrow w=\varphi(z)=\frac{\alpha z+\beta}{\gamma z+\sigma}
\end{gathered}
$$


where $\alpha, \beta, \gamma, \sigma \in \mathbb{C}$ are complex numbers which satisfy $\alpha \cdot \sigma-\gamma \cdot \beta \neq 0$ for $A=\left[\begin{array}{ll}\alpha & \beta \\ \gamma & \sigma\end{array}\right] . \Psi$ denotes the set of all inhomogeneous linear transformations.

The inverse transformation of the $\varphi \in \psi$ is also defined as

$$
\begin{aligned}
& \varphi^{-1}: \mathbb{C}^{*} \rightarrow \mathbb{C}^{*} \\
& w \rightarrow z=\varphi^{-1}(w)=\frac{\sigma w-\beta}{-\gamma w+\alpha} .
\end{aligned}
$$

The set $\varphi$ is a group under the combination of the functions. Also, it is holomorphic for $z \in \mathbb{C}$ with $\varphi \in \Psi, \omega \neq-\frac{\alpha}{\gamma} . \varphi$ transforms on itself to the set of circles on $w$.

Let $A$ be the group of reversible 2 by 2 matrices on $\mathbb{C}$.If $A \in \wp$ then the determinant of the matrix

$$
A=\left[\begin{array}{ll}
\alpha & \beta \\
\gamma & \delta
\end{array}\right] \text { is }|A|=\alpha \delta-\beta \gamma \neq 0
$$

where $\alpha, \beta, \delta, \gamma \in \mathbb{C}$.

Let $\varphi$ also be the set of invertible linear maps of $\mathbb{C}^{2}$ onto itself, which we refer to as homogeneous linear transformations.

The transformation

$$
\varphi_{A}: \mathbb{C}^{2} \rightarrow \mathbb{C}^{2}, z \rightarrow w=A . z
$$

belongs to $\varphi$ for any $A \in \wp$ where $A . z$ is the matrix product of $A$ by column $z=\left[\begin{array}{l}\omega_{1} \\ \omega_{2}\end{array}\right] \in \mathbb{C}^{2}$.

From here,

$$
w=A \cdot z=\left[\begin{array}{ll}
\alpha & \beta \\
\gamma & \delta
\end{array}\right]\left[\begin{array}{l}
\omega_{1} \\
\omega_{2}
\end{array}\right]=\left[\begin{array}{l}
\alpha \omega_{1}+\beta \omega_{2} \\
\gamma \omega_{1}+\delta \omega_{2}
\end{array}\right]=\left[\begin{array}{l}
w_{1} \\
w_{2}
\end{array}\right] \text { is obtained. }
$$

$\phi: \wp \rightarrow \Psi, A \rightarrow \bar{A}: z \rightarrow w=\bar{A}(z)=\frac{\alpha z+\beta}{\gamma z+\delta} \quad$ is a homomorphism with the kernel of $\mathbb{C}^{*}=\mathbb{C} \backslash\{0\}$.

If $\phi$ restricts itself to the group $\wp^{*}$ of unimodular matrices 


$$
A=\left[\begin{array}{ll}
\alpha & \beta \\
\gamma & \delta
\end{array}\right] \in \wp^{*},|A|=\alpha \delta-\beta \gamma=1
$$

then the kernel of $\phi$ is $\{ \pm I\}$ and $\wp / \mathbb{C}^{*} \cong \Psi, \wp^{*} /\{ \pm I\} \cong \Psi$.

A homogeneous modular transformation is a homogeneous linear transformation with rational integers as elements and a determinant equal to one. It forms a group called a homogeneous modular group. This group

$$
\Gamma:=\left\{\left[\begin{array}{ll}
a & b \\
c & d
\end{array}\right], a, b, c, d \in \mathrm{Z}, a d-b c=1\right\}
$$

is isomorphic to unimodular matrix group.

The homomorphism $\phi$ shows the inhomogeneous $\bar{\Gamma}:=\{\bar{A} \mid A \in \Gamma\}$ modular group.

Here if $A=\left[\begin{array}{ll}a & b \\ c & d\end{array}\right] \in \Gamma$ then $\bar{A}: z \rightarrow \frac{a z+b}{c z+d}$.

The components inhomogeneous modular transformations are referred to as $\bar{A} \in \bar{\Gamma}$. They retain the upper half plane $\kappa$, the real axis $\mathbb{R}$, and the set of rational numbers $\mathbb{Q}$.

Given by isomorphism

$$
\Gamma /\{ \pm I\} \cong \bar{\Gamma}
$$

describes the relationship between $\Gamma$ and $\bar{\Gamma}$.

If $A=\left[\begin{array}{ll}a & b \\ c & d\end{array}\right] \in \Gamma$ and $\operatorname{tr}(A)=a+d$ then the characteristic polynomial is

$$
\theta_{A}(\chi)=\operatorname{det}\left[\begin{array}{cc}
a-\chi & b \\
c & d-\chi
\end{array}\right]=\chi^{2}-\operatorname{tr}(A) \chi+1 \text { It has two roots such that }
$$

$$
\lambda_{1,2}=\frac{a+d}{2} \pm \frac{1}{2} \sqrt{(a+d)^{2}-4}
$$

Clearly $\lambda_{1} \lambda_{2}=1$.

The following special matrices belonging to $\Gamma$ occur

$$
B:=\left[\begin{array}{ll}
1 & 1 \\
0 & 1
\end{array}\right], D:=\left[\begin{array}{cc}
0 & -1 \\
1 & 0
\end{array}\right], E:=\left[\begin{array}{ll}
1 & 0 \\
1 & 1
\end{array}\right] \text {. }
$$


The corresponding mappings are given by

$$
B z=z+1, D z=-\frac{1}{z}, W z=\frac{z}{z+1}
$$

We note that, for any $k \in \mathbb{Z}$

$$
B^{k}=\left[\begin{array}{ll}
1 & k \\
0 & 1
\end{array}\right]
$$

Moreover,

$$
D^{2}=-I, P^{3}=-I,
$$

and $P:=D B=\left[\begin{array}{cc}0 & -1 \\ 1 & 1\end{array}\right]$

Also, $P^{2}=\left[\begin{array}{cc}-1 & -1 \\ 1 & 0\end{array}\right]$ and $E=B D B$

The mappings $D$ and $P$ have periods 2 and 3 respectively.

$R$ is any commutative ring and $R^{*}$ is the multiplicative group of the inverse elements of the $R$ ring. The group of the matrices in form $A=\left[\begin{array}{ll}a & b \\ c & d\end{array}\right]$ with determinant equal to 1 is shown as $S \varphi_{2}$ where $a, b, c, d \in R$ and $\operatorname{det} A=a d-b c \in R^{*}$.

Here $R=\mathbb{R}, Z$ or $Z_{n}$ can be taken.

The modular group denoted by $S \varphi_{2}(\mathbb{Z})$ is the subgroup of $S \varphi_{2}(\mathbb{R})$ whose elements are defined in the form

$$
\Gamma:=\left\{\left[\begin{array}{ll}
a & b \\
c & d
\end{array}\right], a, b, c, d \in \mathrm{Z}, a d-b c=1\right\}
$$

by $2 \times 2$ matrices with integer coefficients and determinant equal to 1 .

Let $H=\{z \in \mathbb{C}: \operatorname{Im} z>0\}$. 
We write $\varphi=\left[\omega_{1}, \omega_{2}\right]$ if $\omega_{1}, \omega_{2}$ is a basis of the lattice $\varphi$. We always assume that $\tau=\frac{\omega_{1}}{\omega_{2}} \in H$. Otherwise it is $\tau=\frac{\omega_{2}}{\omega_{1}} \in H$ and we can write $\varphi=\left[\omega_{2}, \omega_{1}\right]$.

The set of all transformations of the form $F: \bar{A}=A(\tau)=\frac{a \tau+b}{c \tau+d}$ is called the inhomogeneous modular transformations (Apostol, 1976).

\section{Resarch Findings}

Let $\Gamma=S \varphi_{2}(\mathbb{Z})$. We define $\Gamma_{Z^{+}}\left(\right.$or $\left.\Gamma\left(\mathbb{Z}^{+}\right)\right)$for each positive integer $\mathbb{Z}^{+}$to be the subgroup of the modular group $\Gamma$ consisting of those matrices satisfying the condition

$$
\left[\begin{array}{ll}
a & b \\
c & d
\end{array}\right] \equiv I\left(\operatorname{Mod} \mathbb{Z}^{+}\right) \text {for unit matrix } I=\left[\begin{array}{ll}
1 & 0 \\
0 & 1
\end{array}\right] \text {. }
$$

In other words,

$a \equiv d \equiv 1\left(\operatorname{Mod} \mathbb{Z}^{+}\right)$and $c \equiv d \equiv 0\left(\operatorname{Mod} \mathbb{Z}^{+}\right)$(Wilson, 1987)

The congruence subgroup of level $N$ is referred to as $\Gamma_{N}$. Inhomogeneous modular transformations

$$
F_{1}: \bar{S}=S(\tau)=-\frac{1}{\tau}
$$

for matrix $S=\left[\begin{array}{cc}0 & -1 \\ 1 & 0\end{array}\right] \in \Gamma$,

$$
F_{2}: \bar{U}=U(\tau)=-\frac{1}{\tau+1}
$$

for matrix $U=\left[\begin{array}{cc}0 & 1 \\ -1 & -1\end{array}\right] \in \Gamma$ and $T=S U$

generate $\bar{\Gamma}$, has proposition $F_{1}^{2}=F_{2}^{3}=I(\tau)$

In fact,

$$
\begin{gathered}
F_{1}: \bar{S}=\mathrm{S}(\tau)=-\frac{1}{\tau} \\
\Rightarrow{F_{1}}^{2}=S(\tau) o S(\tau)=I(\tau)
\end{gathered}
$$




$$
\begin{gathered}
F_{2}: \bar{U}=U(\tau)=-\frac{1}{\tau+1} \\
\Rightarrow F_{2}{ }^{3}=U(\tau) o U(\tau) o U(\tau)=I(\tau)
\end{gathered}
$$

We'll look at $F_{1}, F_{2}$ and $F_{3}$ transformations. Transformation $F_{1}$ is the reflection in the imaginary axis for $\tau=i$. Transformation $F_{2}$ is a reflection in region $\operatorname{Re} F_{2}=-\frac{1}{2}$ for $\tau=i$. Transformation $F_{3}$, which is $F_{3}: \bar{R}=R(\tau)=\tau$ for matrix $R=-S U$ is a reflection in the unit circle $|\tau|=1$.

Now we prove the following theorem with a different matrix.

Theorem: The two matrices $T=\left[\begin{array}{ll}1 & 1 \\ 0 & 1\end{array}\right]$ and $S=\left[\begin{array}{cc}0 & -1 \\ 1 & 0\end{array}\right]$ are used to generate $\Gamma$ modular group.

Proof: By taking the matrix $A$

$$
A=\left[\begin{array}{ll}
a & b \\
c & d
\end{array}\right]=\left[\begin{array}{cc}
1 & n \\
2 & 2 n+1
\end{array}\right] \in \Gamma
$$

$A$ is written as a product of powers of $T$ and $S$. Only the first power of $S$ will occur since $S^{2}$ $=I$. Therefore we obtain

$$
A T^{n}=\left[\begin{array}{cc}
1 & n \\
2 & 2 n+1
\end{array}\right] \cdot\left[\begin{array}{cc}
1 & n \\
0 & 1
\end{array}\right]=\left[\begin{array}{cc}
1 & 2 n \\
2 & 4 n+1
\end{array}\right]
$$

From (3.1), $\operatorname{det}\left(A T^{n}\right)$ is one. By choosing suitable $n$ values, for example, taking $n=0$

$$
A T^{0}=\left[\begin{array}{ll}
1 & 0 \\
2 & 1
\end{array}\right]=A^{0} \in \Gamma
$$

Next, multiplying both sides of the (3.1) from the right by $S$

$$
A T^{n} S=\left[\begin{array}{cc}
1 & 2 n \\
2 & 4 n+1
\end{array}\right]\left[\begin{array}{cc}
0 & -1 \\
1 & 0
\end{array}\right]=\left[\begin{array}{cc}
2 n & -1 \\
4 n+1 & -2
\end{array}\right]
$$

Letting $n=0$ 


$$
A T^{0} S=\left[\begin{array}{ll}
0 & -1 \\
1 & -2
\end{array}\right]
$$

Similarly, by multiplying an appropriate power of $T$, that is $T^{2}$

$$
\begin{aligned}
A T^{n} S T^{2} & =\left[\begin{array}{cc}
2 n & -1 \\
4 n+1 & -2
\end{array}\right]\left[\begin{array}{ll}
1 & 2 \\
0 & 1
\end{array}\right] \\
& =\left[\begin{array}{cc}
2 n & 4 n-1 \\
4 n+1 & 8 n
\end{array}\right]
\end{aligned}
$$

gives us this matrix.

Thus choosing $n=0$, we obtain

$$
A T^{0} S T^{2}=\left[\begin{array}{cc}
0 & -1 \\
1 & 0
\end{array}\right]=S \in \Gamma
$$

The matrix $\left[\begin{array}{cc}2 n & 4 n-1 \\ 4 n+1 & 8 n\end{array}\right]$ is made by product of powers of $T$ and $S$, which is $S=\left[\begin{array}{cc}0 & -1 \\ 1 & 0\end{array}\right]$ for $n=0$.

Solving for $A$, we can obtain

$$
\begin{aligned}
T^{k} S & =\left[\begin{array}{cc}
1 & k \\
0 & 1
\end{array}\right] \cdot\left[\begin{array}{cc}
0 & -1 \\
1 & 0
\end{array}\right]=\left[\begin{array}{cc}
k & -1 \\
1 & 0
\end{array}\right] \\
& \Rightarrow T^{k} S T^{2}=\left[\begin{array}{cc}
k & 2 k-1 \\
1 & 2
\end{array}\right] \\
& \Rightarrow T^{k} S T^{2} S=\left[\begin{array}{cc}
2 k-1 & -k \\
2 & -1
\end{array}\right] \\
& \Rightarrow T^{k} S T^{2} S T=\left[\begin{array}{cc}
2 k-1 & k-1 \\
2 & 1
\end{array}\right] \\
& \Rightarrow T^{n+1} S T^{2} S T=\left[\begin{array}{cc}
2 n+1 & n \\
2 & 1
\end{array}\right]
\end{aligned}
$$

where $k=n+1$. 
$T^{n+1} S T^{2} S T$ matrix produced of powers of $S$ and $T$ is equivalent to $A=\left[\begin{array}{ll}1 & 0 \\ 2 & 1\end{array}\right]$ matrix for $n=0$. We get $A=T^{n+1} S T^{2} S T$.

It is sufficient to consider the matrix $\left[\begin{array}{ll}a & b \\ c & d\end{array}\right] \in \Gamma$ to prove the theorem in general. On $c$, we use induction in the following way:

a) If $c=0$ then $a d=1$ so $a=d= \pm 1$.

In this case,

$$
A=\left[\begin{array}{ll}
a & b \\
c & d
\end{array}\right]=\left[\begin{array}{cc} 
\pm 1 & b \\
0 & \pm 1
\end{array}\right]= \pm\left[\begin{array}{cc}
1 & \pm b \\
0 & 1
\end{array}\right]= \pm T^{ \pm b} \quad \text { By taking } \pm b=q_{0} \text { then } A=T^{q_{0}} .
$$

Therefore, $A$ is a power of matrix $T$.

b) If $c=1$ then $a d-b=1$ so $b=a d-1$ when the matrix $A$ is rewritten as

$$
\begin{aligned}
A=\left[\begin{array}{ll}
a & b \\
c & d
\end{array}\right] & =\left[\begin{array}{cc}
a & a d-1 \\
1 & d
\end{array}\right] \\
& =\left[\begin{array}{ll}
1 & a \\
1 & d
\end{array}\right] \cdot\left[\begin{array}{cc}
0 & -1 \\
1 & 0
\end{array}\right] \cdot\left[\begin{array}{ll}
1 & d \\
0 & 1
\end{array}\right] \\
& =T^{a} S T^{d} .
\end{aligned}
$$

According to the induction theorem, the last matrix is a product of $T$ and $S$ powers, so $A$ is as well. This concludes the proof of the theorem. As a result, the theorem was proved using the general matrix $\left[\begin{array}{cc}1 & n \\ 2 & 2 n+1\end{array}\right]$ rather than the special matrix $\left[\begin{array}{cc}4 & 9 \\ 11 & 25\end{array}\right]$ used by Apostol previously.

\section{Fundamental Regions.}

The upper half-plane generated by the extended upper half-plane formed by the rational points of the real axis and imaginary axis $i \infty$ is described by $\kappa^{x}$ and the upper half- plane is $\kappa$ and its variable is $\tau$.

Let $\Gamma^{x}=(T(\tau), S(\tau))=(\bar{T}, \bar{S}) \subset \bar{\Gamma}$

be the group generated by $T(\tau)$ and $S(\tau)$. There is a point equivalent to $\tau$ under $\kappa^{x}$ which has a representative in

$\mathfrak{I}_{\bar{\Gamma}}=\left\{\tau: \tau \in \kappa,|\operatorname{Re} \tau| \leq \frac{1}{2},|\tau| \geq 1\right\} \cup\{i \infty\}$ 
for every $\tau \in \kappa$ since the transformations $\bar{T}=T(\tau)$ and $\bar{S}=S(\tau)$ already line in $\kappa^{x}$ (Lehner, 1965).

Now suppose that $A=\left[\begin{array}{ll}a & b \\ c & d\end{array}\right] \in \Gamma$ is given and complex number $\tau$ such that $\operatorname{Im} \tau>0$, then corresponding to $\bar{A}=A(\tau)$ there is a $\bar{B}=B(\tau) \in \Gamma^{x}$ with $\overline{B A}=B A(\tau) \in \mathfrak{I}_{\bar{\Gamma}}$ and $B A(\tau)=\mathrm{I}$ ( $\tau)$ so $A(\tau)=B^{-1}(\tau) \in \Gamma^{x}$, and $\Gamma^{x}=\bar{\Gamma}$.

Thus, we have a proof for the assertion that $T(\tau), S(\tau)$ and $R(\tau)$ generate $\bar{\Gamma}$.

The image of an element $\tau \in \mathfrak{I}_{\bar{\Gamma}}$ distinct from $r, i$ and $i \infty$ defines tessellation of the upper half-plane as an inhomogeneous modular transformation. As a result, we can deduce that any element $\tau$ of the expanded upper half-plane $\kappa^{x}$, different from $r, i$ and $i \infty$, appears exactly once as the image under $\bar{\Gamma}$ of an element in $\mathfrak{J}_{\bar{\Gamma}}$ (Swinnerton-Dyer and Birch., 1975). Obviously, the points equivalent to $i$ are covered twice, the points equivalent to $r$ are covered twice, the points equivalent to $i \infty$ are covered an infinite number of times. For arbitrary $U \in \Gamma$ , we understand the image of $\mathfrak{I}_{\bar{\Gamma}}$ under $\bar{U}=U(\tau)$ Clearly, $\mathfrak{I}_{\bar{\Gamma}(U(\tau))}$ is a fundamental region because of $U(\tau) \in \bar{\Gamma}$ for $U \in \Gamma$ and

$$
\mathfrak{I}_{\bar{\Gamma}(U(\tau))}=\bar{U}\left(\mathfrak{I}_{\bar{\Gamma}}\right)
$$

The upper half-plane can be divided into modular triangular regions, which are made up of that cover the upper half-plane. There are three modular triangular regions adjacent to each modular triangular region. For general matrix $U=\left[\begin{array}{ll}a & b \\ c & d\end{array}\right] \in \Gamma$, we will be following three modular triangular regions that are adjacent to each other.

a) $U T U^{-1}(\tau)=\overline{U T U^{-1}}\left(\mathfrak{I}_{\bar{\Gamma}}\right)$ for matrix

$$
U T U^{-1}=\left[\begin{array}{cc}
1-a c & a^{2} \\
-c^{2} & 1+a c
\end{array}\right] \in \Gamma
$$

b) $U T^{-1} U^{-1}(\tau)=\overline{U T^{-1} U^{-1}}\left(\mathfrak{J}_{\bar{\Gamma}}\right)$ for matrix $U T^{-1} U^{-1}=\left[\begin{array}{cc}1+a c & -a^{2} \\ c^{2} & 1-a c\end{array}\right] \in \Gamma$

c) $U S U^{-1}(\tau)=\overline{U S U^{-1}}\left(\mathfrak{J}_{\bar{\Gamma}}\right)$ for matrix $\quad U S U^{-1}=\left[\begin{array}{cc}a c+b d & -a^{2}-b^{2} \\ c^{2}+d^{2} & -a c-b d\end{array}\right] \in \Gamma$ 


\section{Results}

Using the modular group conditions, we investigate the generations of inhomogeneous modular groups and prove the theorem about modular group generations using a different approach.

\section{References}

Apostol, T. M. (1976). "Modular functions and Dirichlet series in number theory”, Graduate texts in mathematics, Springer-Verleng, New York.

L. Wilson., G. 1987. “A Family of Modular Functions Arising from the Theta Function”, Proc, London math. Soc. (3), 55.

Lehner, J. (1965). “A short course in automorphic functions”, University of Maryland.

Swinnerton-Dyer, H.P.F. and Birch B. J. (1975). "Modular functions of one variable IV", Lecture notes in Math., Springer Verlag, Berlin-Heidelberg New York, 476, 2-32. 